\title{
The therapeutic needs of psychiatric in-patients with psychosis: A qualitative exploration of patient and staff perspectives
}

\author{
Lisa Wood, Claire Williams, Jo Billings and Sonia Johnson
}

\section{Background}

Concerns are recurrently expressed that the therapeutic content of in-patient care is limited and lacking clear guidance. The perspectives of patients and staff regarding therapeutic priorities for psychiatric in-patient care have been little explored and compared.

\section{Aims}

The aim of this study was to examine patient and staff perspectives on the care priorities of psychiatric in-patients with psychosis.

\section{Method}

We recruited 12 in-patients with psychosis and 12 multidisciplinary team staff. All participants undertook a semi-structured interview examining their perspectives on the therapeutic needs of people with psychosis during admission. Interviews were transcribed verbatim and thematic analysis conducted.

\section{Results}

Three superordinate themes arose from patient interviews: 'the importance of considering social circumstances and trauma', 'managing the intra- and interpersonal impact of psychosis' and 'lack of control and collaboration in care' and three from staff interviews: 'multidisciplinary facilitators of care', 'treating complexity and incorporating social factors' and 'restrictive practices preventing quality care provision'. Comparison of patient and staff themes identified unmet needs in addressing social marginalisation, trauma and distress, and the importance of collaborative treatment process and inclusion of spirituality.

\section{Conclusions}

There are gaps between staff and patient perspectives on important priorities for in-patient care that may help explain persistent patient dissatisfaction with in-patient care. Findings suggest the need for coproduced work to develop and test interventions that address broader therapeutic priorities.

\section{Declaration of interest}

None.

Keywords

Psychotic disorders; qualitative research; in-patient treatment.

\section{Copyright and usage}

(C) The Royal College of Psychiatrists 2019. This is an Open Access article, distributed under the terms of the Creative Commons Attribution-NonCommercial-NoDerivatives licence (http://creativecommons.org/licenses/by-nc-nd/4.0/), which permits noncommercial re-use, distribution, and reproduction in any medium, provided the original work is unaltered and is properly cited. The written permission of Cambridge University Press must be obtained for commercial re-use or in order to create a derivative work.
Psychosis is judged to be one of the top 25 causes of worldwide disability. ${ }^{1}$ It is one of the mental illnesses with the most impact on life and associated with poorest lifelong outcomes, and has the greatest variation in access to evidence-based informed care. ${ }^{2}$ Up to two-thirds of the current psychiatric hospital population are experiencing psychosis internationally. ${ }^{3}$ Compulsory admissions to a psychiatric hospital are high, with people experiencing psychosis being the most likely group to be forcibly detained. ${ }^{4,5}$ Psychiatric in-patient care is the most expensive form of care ( $£ 437$ per day per bed) compared with all other out-patient care alternatives, with costs continuing to increase. ${ }^{6}$ Problematically, there continues to be a lack of clarity regarding the purpose and function of psychiatric hospital care. ${ }^{7}$ Patients and staff report that the focus of in-patient care is excessively narrow, with too much focus on reducing risk and containing a mental health crisis, and that there is a lack of developed therapeutic models for addressing a wider range of psychosocial needs. ${ }^{7}$ Patients also report ongoing dissatisfaction with in-patient care because of the lack of therapeutic activity and poor relationships with staff. ${ }^{8}$

Improving in-patient care is a central issue for health services, as outlined by the recent UK Francis Report. ${ }^{9}$ The influential Five Year Forward View UK government report states that the complexity of psychiatric in-patients is worsening and forced admissions are also continuing to rise, indicating a systematic failure to provide effective crisis care for in-patients. ${ }^{10}$ The document argues that in-patient wards are often not safe, therapeutic or conducive to recovery. A recent qualitative systematic review of patient perspectives has demonstrated that psychiatric in-patient care is not meeting their requirements, and that further research is required to understand the disparity between patient and staff priorities. ${ }^{8}$ This study aimed to examine patient and staff perspectives on the care priorities of people experiencing psychosis within a psychiatric inpatient setting. More specifically, it aimed to examine (a) the care priorities of in-patients experiencing psychosis from the patient perspective; (b) the care priorities for in-patients experiencing psychosis from the in-patient staff perspective; and (c) the key similarities and differences in perceived care priorities. A qualitative semi-structured approach was undertaken to allow for the exploration of subjective experiences of care priorities.

\section{Method}

\section{Design}

Qualitative semi-structured interviews were conducted with psychiatric in-patients and staff to examine the care priorities of inpatients experiencing psychosis. The research followed guidance from the 32-item Consolidated Criteria for Reporting Qualitative Research ${ }^{10}$ to increase methodological quality and reporting. The authors assert that all procedures contributing to this work comply with the ethical standards of the relevant national and institutional committees on human experimentation and with the Helsinki Declaration of 1975 , as revised in 2008. All procedures involving human patients were approved by the Health Research 
Authority (IRAS 222917) and NHS ethical approval was given (17/WM/2055).

\section{Participants}

All potential participants were recruited from an outer London psychiatric in-patient unit. A total of 12 psychiatric in-patients and 12 multidisciplinary team (MDT) in-patient staff members were recruited for this study. Patient participants were recruited via in-patient ward staff to ensure patients did not feel coerced or pressured into taking part. Sample size was determined by thematic analysis sample size guidelines and considering the anticipated theme prevalence and instances. ${ }^{11}$ All potential participants approached met all eligibility criteria and were able to take part in the study. No potential participants refused to take part. Purposive sampling was undertaken to gather a diverse patient group. Factors that were taken into consideration were, representation from both white and minority ethnic backgrounds, including both firstepisode and recurrent psychosis, and varied numbers of admissions. For staff, it was ensured that participants were from differing professional backgrounds (nursing, occupational therapy, psychiatry).

Patient participants were included if they were (a) aged 18 and above; (b) currently admitted to an acute psychiatric in-patient unit (on section or informally); (c) met criteria for a schizophrenia-spectrum diagnoses or threshold for early intervention services (selfreported); (d) had capacity to give informed consent; (e) able to complete the study in English. Participants were excluded if they were (a) non-English speaking (because of translation costs and the impact on qualitative analysis); (b) had an acquired brain injury or substance misuse judged to be the acute cause of their psychotic experience; (c) lacking the capacity to give informed consent (assessment through comprehension of participant information sheet with researcher); (d) experiencing severe thought disorder; (e) has a primary presentation of depression or bipolar disorder and that psychotic experiences are secondary. Staff participants were included if they were (a) a qualified member of clinical staff (psychiatric nurse, doctor, occupational therapist); and (b) working in a psychiatric in-patient MDT.

\section{Data collection}

Semi-structured interviews were chosen to collect data as they allow for the systematic and comprehensive exploration of the topic area. This methodology ensured that all participants, particularly patients were able to meaningfully voice their perspectives in a confidential and safe space. All interviews were conducted by L.W., an experienced qualitative researcher and clinical psychologist who works clinically in the recruiting psychiatric in-patient setting site. L.W. did not know any of the patient participants, and had only worked with one of the staff participants, prior to the commencement of the study. Two semi-structured interview schedules were developed to examine psychiatric in-patients' and staffs' perspectives on care priorities of current psychiatric in-patients experiencing psychosis. Both interview schedules asked about their in-patient experiences, priorities for care, recovery goals, treatment availability and considerations for how care could be improved. Both interview schedules were developed by consulting relevant literature, ${ }^{7,8}$ and also in consultation with a patient and public advisory panel and with in-patient staff. All interviews took place on the psychiatric in-patient ward.

\section{Data analysis}

Patient and staff data were analysed separately, and overall themes were compared once each analysis was complete. Interviews were recorded and transcribed verbatim, coded and categorised using
NVIVO 11 computer programme. ${ }^{12}$ Thematic analysis was used to analyse data and a critical-realist epistemological position was adopted. ${ }^{13}$ Latent themes were extracted and an inductive approach to data analysis was undertaken. A core concept of thematic analysis is that the analyst should become immersed in the data. Interviews were listened to and the transcripts read a number of times. Analysis was conducted in three stages:

(a) free line-by-line coding of each interview with codes being extracted if considered to represent patient care needs;

(b) codes were translated across interviews and related codes were grouped together;

(c) 'analytical' themes were developed and agreed upon in supervision.

Once analysis was complete, a random selection of $25 \%$ of participants were invited back to give feedback on the results. They were presented with the analysis and asked for their opinions on the accuracy of interpretation. Finally, the analytical themes for patients and staff were then compared in order to identify similarities and differences.

\section{Results}

A total of 12 patients and 12 MDT staff member participants took part in the study. Participant and staff demographics can be found in Table 1. Patient interviews lasted on average $37.58 \mathrm{~min}$ $($ s.d. $=12.01)$ and staff interviews $46.17 \mathrm{~min}($ s.d. $=10.07)$.

\section{Patient priorities}

We identified 3 superordinate themes and 13 subordinate themes in the analysis of patient transcripts. These are outlined in Appendix 1 and are supported by participant quotes. Quotes are numbered so they can be cross-referenced with Appendix 1. The participant sample had an average age of 41.33 (s.d. $=12.04$ ) and average number of admissions of nine (s.d. = 13.41), were predominantly men $(n=11,91.7 \%)$ and under section of the Mental Health Act (MHA) $(n=11,91.7 \%)$, unemployed $(n=9,75 \%)$, of religious faith $(n=9,75 \%)$, and diagnosed with schizophrenia $(n=7,58.3 \%)$.

\section{The importance of considering social circumstances and trauma}

The first superordinate theme was the importance of including patients' social circumstances, trauma and relationships within care planning, as they were important in understanding the patients' reasons for admission. Social difficulties and trauma were cumulative with patients experiencing multiple ones in their lives.

\section{Overwhelming social issues}

Feeling excluded from society with poor life opportunities were described by participants as contributors to admission and required prioritising in their care plans (Appendix 1, quote 1). Participants described prioritising social issues such as lack of accommodation, financial hardship and employment difficulties. Stigma and discrimination were often considered as causes of these difficulties, and the gravity of these issues were not always fully comprehended by in-patient staff.

\section{Trauma and grief contributing to the crisis}

Participants experienced multiple distressing traumatic experiences and grief, which the participants thought contributed to their mental health breakdown and crisis (Appendix 1, quote 2). These experiences included the death of a loved one, violence, abuse and 


\begin{tabular}{|c|c|}
\hline Characteristic & Value \\
\hline \multicolumn{2}{|l|}{ Patient participants } \\
\hline Age, mean (s.d.) & $41.33(12.04)$ \\
\hline Number of admissions, mean (s.d.) & $9(13.41)$ \\
\hline Length of current admission, days: mean (s.d.) & $24.72(12.63)$ \\
\hline Length of psychosis experiences, years: mean (s.d.) & $18.27(10.32)$ \\
\hline \multicolumn{2}{|l|}{ Gender, $n$} \\
\hline Men & 11 \\
\hline Women & 1 \\
\hline \multicolumn{2}{|l|}{ Ethnicity, $n$} \\
\hline White (British and European) & 5 \\
\hline Black (British, African and Caribbean) & 3 \\
\hline Asian (Indian) & 1 \\
\hline Other & 3 \\
\hline \multicolumn{2}{|l|}{ Education level, $n$} \\
\hline Secondary & 8 \\
\hline Further & 2 \\
\hline Higher & 2 \\
\hline \multicolumn{2}{|l|}{ Employment status, $n$} \\
\hline Unemployed & 9 \\
\hline Employed & 2 \\
\hline Volunteering & 1 \\
\hline \multicolumn{2}{|l|}{ Marital status, $n$} \\
\hline Single & 10 \\
\hline Married & 1 \\
\hline Divorced & 1 \\
\hline \multicolumn{2}{|l|}{ Religious beliefs, $n$} \\
\hline Christianity & 5 \\
\hline Islam & 3 \\
\hline None & 3 \\
\hline Other & 1 \\
\hline \multicolumn{2}{|l|}{ Admission status, $n$} \\
\hline Involuntary & 11 \\
\hline Voluntary & 1 \\
\hline \multicolumn{2}{|l|}{ Diagnosis, $n$} \\
\hline Schizophrenia & 7 \\
\hline Bipolar disorder (with psychosis) & 4 \\
\hline First-episode psychosis & 1 \\
\hline \multicolumn{2}{|l|}{ In-patient staff participants } \\
\hline Age, years: mean (s.d.) & $38.18(10.40)$ \\
\hline Length of mental health practice, years: mean (s.d.) & $10.04(7.90)$ \\
\hline Length of inpatient experience, years: mean (s.d.) & $7.08(7.39)$ \\
\hline \multicolumn{2}{|l|}{ Gender, $n$} \\
\hline Men & 8 \\
\hline Women & 4 \\
\hline \multicolumn{2}{|l|}{ Ethnicity, $n$} \\
\hline White (British and European) & 4 \\
\hline Black (British, African and Caribbean) & 6 \\
\hline Asian (Indian) & 1 \\
\hline Chinese & 1 \\
\hline \multicolumn{2}{|l|}{ Professional role, $n$} \\
\hline Occupational therapist & 4 \\
\hline Psychiatric nurse & 3 \\
\hline Psychiatrists & 3 \\
\hline Clinical manager (nurse) & 2 \\
\hline
\end{tabular}

relationship breakdowns. For some, these experiences happened in the past but the ongoing related distress was a contributing factor to their crisis.

\section{Incorporating cultural, religious and spiritual ways of understanding mental health}

Participants described the importance of culture, religion and spirituality in understanding their mental health difficulties (Appendix 1, quotes 3 and 4). This was important to all aspects of their mental health experiences from their understanding of their difficulties (for example believing they were hearing the voice of god) to strategies to cope with them (such as prayer and chaplaincy). Conflict arose in psychiatric in-patient care when patients adopted this framework of understanding as opposed to a medical framework.

\section{Involving family and friends with agreement}

Participants described family and friends playing an important role in gaining access to acute care and for them to be included within their care planning (Appendix 1, quotes 5 and 6). However, some participants acknowledged that family involvement could also be unhelpful so involvement should only occur with their agreement.

\section{Managing the intra- and interpersonal impact of psychosis}

Participants described experiencing psychotic crises, and that the intra- and interpersonal impact that these experiences caused were of primary concern. In other words, coping with the acute personal distress resulting from psychosis, and the impact it had on their relationships, were patients' priority.

\section{Distressing and commanding voices causing harm}

Participants reported severely distressing voices that were derogatory, critical and commanding (Appendix 1, quote 7). It was the high levels of distress and harm that the voices caused that contributed to their crisis. The voices either commanded the person to harm themselves and/or others, or the person self-harmed because they could not cope with the voices. Reducing the harm and distress caused by hearing voices was important to patients.

\section{Paranoia and distressing unusual beliefs - needing to protect myself}

Participants reported experiencing paranoid thoughts, difficulty trusting others and worries about their own safety (Appendix 1, quotes 8 and 9). This often led to participants' withdrawing and protecting themselves through self-harm, suicidality or violence. The feelings of being unsafe also had an impact on their willingness to engage in in-patient care. Participants required more time to build relationships with ward staff and to feel safe while on the ward.

\section{Coping with my crisis the best way I can}

Participants' ways of coping with their crisis often caused them difficulties with their support system (such as family and mental health services) (Appendix 1, quotes 10 and 11). Drug and alcohol use were the most common strategy and a frequent cause of crisis. Participants also reported becoming withdrawn and keeping their problems secret, which meant their difficulties worsened to crisis point before they had access to help. Participants did not always prioritise changing their approaches to coping but consideration of them were important to their in-patient care.

\section{Confusion and emotional distress}

All participants spoke about feeling confused because of their crisis and being admitted onto the ward, the negative impact on their memory and concentration, and significant emotional distress during their admission (Appendix 1, quotes 12 and 13). Reducing these were very important to participants.

\section{Lack of control and collaboration in care}

The final subordinate theme described the perceived obstacles to quality care.

\section{Admission trauma and disorientation}

Many participants experienced their admission as traumatic and disorientating, especially those who were forcibly detained under 
the MHA (Appendix 1, quote 14). Participants reported hazy memories of the first few days and their admission experience. Their route to admission understandably had an impact on how safe they then felt on the ward. Participants reported that staff never asked about their admission experiences and the possible detrimental impact, which was important to them.

\section{Inflexible and unclear treatment}

Participants described finding components of psychiatric in-patient care inflexible and unresponsive to their needs, which resulted in perceived loss of freedom and control, and less willingness to engage in their care plan (Appendix 1, quotes 15 and 16). For example, participants felt that they were not listened to regarding treatment planning. Participants wanted staff to adopt a transparent and communicative approach, which was often absent.

\section{Dominant pharmacological approach to treatment}

Participants described that compulsory pharmacological treatment was the primary treatment offered during admission and that there was less opportunity to engage in psychosocial therapies (Appendix 1, quotes 17 and 18). Non-adherence with medication was often cited as a cause of hospital admission. The perceived helpfulness of pharmacological treatment varied across participants and an informed choice was desired but rarely present.

\section{Poor patient and staff relationships}

Participants placed importance on their relationships with staff and fellow patients (Appendix 1, quotes 19 and 20). Staff relationships were often described within the context of lack of availability and time, which did not allow for meaningful relationships to develop. However, when positive relationships were established they were extremely valued. Regarding patient relationships, participants described violence and aggression between patients that disrupted the quality of their care they received on the ward. However, peer relationships were also an important part of care.

\section{Flexible access to psychosocial treatments}

Participants described wanting to have access to treatments that did not involve medication, however, this was not always available (Appendix 1, quotes 21 and 22). Participants thought that occupational and psychological therapy assisted with their recovery. Not all patients wanted to engage in these activities, and some found discussing their difficulties distressing. Again, informed treatment choice was crucial.

\section{In-patient staff priorities}

The analysis from the psychiatric in-patient staff identified 3 superordinate themes, and 13 subordinate themes. These are outlined in Appendix 2 and are supported by participant quotes. Quotes are numbered so they can be cross-referenced with Appendix 2.

\section{Multidisciplinary facilitators of care}

The first superordinate theme identified was the importance of effective MDT working to provide quality and diversity in care.

\section{Team cohesiveness}

Strong team relationships, where multiple perspectives towards patient care were respected, were described as essential (Appendix 2, quote 1). Participants spoke about the importance of communication and negotiating with one another to implement care. Having strong leadership was integral to a whole team approach.

\section{Biopsychosocial treatments}

All participants spoke about the importance of adopting a biopsychosocial model and offering an equal balance of psychosocial and medical treatments to patients (Appendix 2, quote 2). However, it was acknowledged by staff that a pharmacological approach was dominant. Participants explained that medication was the primary treatment because of the rapid turnover of patients.

\section{Developing therapeutic relationships with patients}

Developing a collaborative relationship with patients to facilitate effective care was a priority (Appendix 2, quotes 3 and 4). A strong collaborative relationship was achieved by staff being available and present, flexible and taking a genuine interest in the patients' needs. Several staff explained the importance of a meaningful relationship based on equal human connection.

\section{Integrated psychological perspectives}

Participants acknowledged the important role of psychology in care planning and supporting the ward staff to deliver in-patient care (Appendix 2, quotes 5 and 6). Participants reported that psychologists offered formulations that helped the team adopt a holistic view of care. Direct clinical interventions, indirect consultation, teaching and training and staff support were valued.

\section{Treating complexity and incorporating social factors}

The second superordinate theme reflected the purpose and functioning of psychiatric in-patient care. Treatment was largely framed from a medical perspective with the primary focus to treat symptoms and reduce risk.

\section{Continued assessment and observation}

All participants discussed the importance of getting a clear understanding of the person's presenting difficulties and reasons for admission to inform care (Appendix 2, quote 7). This was achieved through formal assessment and observation of the patient, particularly early into admission.

\section{Complex and chronic symptom management}

All participants referred to the complex, chronic and acute symptomatology of their patients being extremely challenging to treat (Appendix 2, quotes 8-10). The lack of 'insight' was described by participants as disrupting treatment provision. The high risk of relapse and readmission was discussed as an ongoing challenge for staff. Drug and alcohol use were considered to be significant contributors to the complexity of treating in-patients.

\section{Risk reduction and management}

The management of patient's risk was highlighted as a priority by participants (Appendix 2, quotes 11 and 12). Risk of violence and aggression from patients towards others was considered the dominant risk that the team had to manage. The primary management strategy was the use of medication to reduce risk.

\section{Family and social network support}

Participants acknowledged the importance of including the patients' family and social network within care planning but there appeared to be a blanket approach to their inclusion (Appendix 2, quotes 13 and 14). The lack of social support that patients often have was acknowledged and the need to support them to cultivate networks was highlighted. 


\section{Social problems, functioning and community care planning}

Participants acknowledged the importance of addressing social problems because of the impact they have on patient's mental health (Appendix 2, quotes 15-17). Treatment planning and organising care in their community environment was important. Supporting patients to regain their functioning was also a treatment priority.

\section{Restrictive practices preventing quality care provision}

The final superordinate theme, highlighted by all professional participants, was regarding the barriers and challenges of implementing psychiatric in-patient treatment.

\section{Restrictive and rigid treatment}

Participants explained how the ward treatment was restrictive and rigid, despite them wanting to offer a more therapeutic approach, as many patients were under section of the MHA with restricted leave, staff described that patients often feel powerless (Appendix 2, quotes 18 and 19). Participants also described the conflicting treatment goals between staff and patients resulting from forced treatment.

\section{Lack of resources and confined environment}

Participants described the impact that lack of resources had on providing the psychiatric in-patient care they would like to (Appendix 2, quotes 20 and 21). The primary difficulty was the lack of time for space and reflection to process the difficult decisions they are making on a day-to-day basis. Another common example was the lack of psychology provision available on the wards.

\section{Engagement difficulties}

Staff reported difficulties engaging patients on the ward (Appendix 2, quotes 22 and 23). A main reason was the fluctuating degrees of wellness experienced by patients that made it particularly difficult for them to engage in psychosocial interventions. Staff also spoke about idiosyncratic barriers that made it difficult to develop relationships with patients, such as lack of insight, violence and aggression.

\section{Lack of safety}

All participants acknowledged the lack of safety present on psychiatric wards as a barrier to providing therapeutic care (Appendix 2, quotes 24 and 25). The ward environment was described as distressing for patients, worsening their mental health. However, participants acknowledged the importance of striving for safety within the psychiatric in-patient environment.

\section{Key differences in patient and staff perspectives}

The analysis has identified key areas of disparity between staff and patient priorities, which are explored here.

(a) Collaboration, meaningful treatment choice and control.

(b) Addressing social marginalisation.

(c) Positive therapeutic staff-patient relationships.

(d) Patient-led family involvement.

(e) Reducing distress rather than symptom reduction.

(f) Incorporation of trauma (historical, recent and admission trauma) into care planning.

(g) Including spiritual, cultural and religious frameworks into care.

\section{Collaboration, meaningful treatment choice and control}

It was important to all patient participants to have collaborative and meaningful choice regarding their treatment options. Some appreciated medical treatments, while others did not. The same was found for occupational and psychological therapies. Both patients and staff acknowledged the limitations of the in-patient ward, which restricted the ability for patients to have truly meaningful treatment choice.

\section{Addressing social marginalisation}

Staff spoke about the importance of improving patient's social functioning, which was important, but this did not appear to capture the breadth of social problems patients faced and difficulty integrating meaningfully within society. Furthermore, the consideration of social issues, such as, employment and functioning, appeared prescriptive and did not address the idiosyncratic nature of these priorities.

\section{Positive therapeutic staff-patient relationships}

Despite staff-patient relationships being prioritised by both groups, it was evident from patient analysis that positive relationships with staff were not experienced during admission. Staff acknowledged barriers to having therapeutic relationships with patients, which may be why this was not achieved.

\section{Patient-led family involvement}

Staff discussed the importance of integrating family involvement within patient care as a priority. However, as identified in the patient analysis, often complex family dynamics were at play, and patients did not always experience their families as supportive. Patient consent and consultation about if, and how, they want their family involved in their care was imperative.

\section{Reducing distress rather than symptom and risk reduction}

Staff participants spoke about the primary purpose of in-patient care being to reduce symptoms and risk reduction. Patients however, did not always prioritise reducing symptoms per se, and appeared to rather focus on the distress resulting from symptoms. This was the same for managing risk and harm.

Incorporation of trauma (historical, recent and admission trauma) into care planning

What was absent from the staff data was the acknowledgment of addressing and caring for trauma experiences, both historical and recent, including trauma that has resulted from a distressing admission experience. Patients often described this as a contributing factor to their admission.

\section{Including spiritual, cultural and religious frameworks into care}

Spirituality, culture and religion was also omitted from the staff analysis, whereas it was prioritised by patients as an important framework to understand their difficulties, as well as manage them. This component of patient care needs to be prioritised by in-patient staff.

\section{Discussion}

\section{Main findings}

This study aimed to examine the care priorities of psychiatric inpatients experiencing psychosis from the perspective of patients and in-patient staff. Three superordinate themes were identified from the analysis of patient data including 'the importance of considering social circumstances and trauma', 'managing the intra- and interpersonal impact of psychosis', and 'lack of control and collaboration in care', and three from the analysis of staff data 'multidisciplinary facilitators of care', 'treating complexity and incorporating social factors' and 'restrictive practices preventing quality care provision'. 


\section{Patient themes}

The three superordinate themes in the patient analysis incorporated experiences that were embedded within their social context and relationships. In particular, the analysis identified that patients experienced a multitude of unresolved traumas and social losses, which precipitated their acute psychotic symptoms and contributed to their reasons for admission. This supports previous research demonstrating the strong cumulative link between experiencing trauma and psychotic experiences. ${ }^{14}$ The understanding of trauma is crucial to effective treatment planning in in-patient care, as identified in other care settings. ${ }^{15}$ Patients also described the importance of their spirituality, culture and religion in relation to their treatment needs, which was not identified by staff participants. Religion and spirituality have been identified as important in the understanding and treatment of psychotic experiences in the inpatient setting as well as with coping with these experiences, ${ }^{16,17}$ and further research needs to be conducted about how we may be able to integrate this into clinical practice. Patients acknowledged that they experienced symptoms of psychosis and harmful behaviours, but these were not prioritised over the intra- and interpersonal distress that psychotic experiences caused. This is in line with the patient community recovery literature that states that recovery can occur despite the presence of symptoms, and that rebuilding self and life are a priority ${ }^{18,19}$ All participants found in-patient treatment to involve a lack of choice and control with experiences described as forced and uncollaborative. Admission was particularly traumatic and often not welcomed, particularly when under the MHA. A recent qualitative paper outlined that freedom and choice, feeling safe and non-paternalistic care from staff were important to improve in-patient care. ${ }^{20}$ There is a clear need to enhance such factors within this setting to address patient concerns identified in this research. ${ }^{21}$

\section{Staff themes}

The staff analysis outlined the importance of having an effective MDT to meet the care priorities of psychiatric in-patients. This was based on team cohesiveness, a biopsychosocial model although acknowledged that the medical model was dominant and strong therapeutic relationships with patients. MDT working is crucial to improving patient outcomes, ${ }^{22}$ which was identified in this study. Staff identified care targets that predominantly focused on the treatment of symptoms and reducing risk, which are the key functions of psychiatric in-patient care. ${ }^{7}$ Staff participants acknowledged the importance of improving social functioning and addressing the social issues, which their patients often face, but that these were secondary to symptoms and risk, and responses to these needs were prescriptive. Staff described restriction in what care can be offered because of a lack of resources. They also described difficulties cultivating a safe environment and good working relationships with patients because of threats of violence, aggression and lack of time. The Kings Fund ${ }^{21}$ demonstrated that as a result of the increased pressure on in-patient beds that the safety and quality of in-patient care is being compromised, and this is supported by these findings.

The overall findings demonstrated some overlap in patient and staff priorities during a psychiatric in-patient admission. This included managing distressing symptoms and improving functioning. Similarly, both raised frustrations regarding the barriers to care, including a lack of time and resources, and restrictive methods of practice. However, there were also key differences in the priorities for psychiatric in-patient care. Despite both parties prioritising positive therapeutic relationships and collaborative care, all key differences in themes identified a clear lack of control and collaboration. Previous research has identified key barriers that prevent collaborative and shared decision-making in in-patient settings including challenges in communication and the physically restrictive hospital environment preventing one-to-one meetings. ${ }^{23}$ It would be important to further investigate how these can be improved to ensure patients feel more in control of their care.

\section{Implications}

There are important clinical implications arising from this research. Patient treatment choice and control is a priority. Collaborative care planning and shared decision-making needs to be integrated into routine in-patient care. More emphasis needs to be placed on supporting patients with social trauma and loss, and religious, cultural and spiritual issues, and ensuring that in-patient staff feel equipped to work collaboratively with these needs. As such, organisational strategies and policies, which underpin the delivery of psychiatric in-patient care, need to emphasise collaborative working practices and place more equal importance on the psychosocial aspects of a patients' presentation. A recent study demonstrated that an intervention aiming to improve collaborative discharge planning using a psychosocial intervention for in-patients was able to increase the use of collaborative recovery planning and improve in-patient satisfaction with their care. ${ }^{24}$ Moreover, a trauma-informed approach to in-patient care may help increase collaboration in care. Trauma-informed approaches have been implemented in the UK National Health Service (NHS) and prioritise a number of key principles relevant to this research. These include transparency and openness, collaborative and coproduced care, avoiding re-traumatisation, developing a safe environment, thoroughly assessing and supporting patients with trauma experiences, and regular collection and evaluation of data relating to use of seclusion and restraint. ${ }^{25}$ Finally, psychiatric in-patient care requires more focus on the distress that can arise from psychotic symptoms and being forcibly detailed under the MHA. There is a clear need to develop interventions that target this that have been coproduced with patients.

\section{Strengths and limitations}

A strength of the research is that it explored the care priorities of patients with psychosis from the perspective of both patients and staff and was able to identify the disparity between perspectives. Moreover, it followed rigorous methods to ensure data collection was of high quality. ${ }^{26}$ Furthermore, a spread of some key demographic factors was achieved, for example age, length of psychosis experiences, number of admissions and ethnicity. There were several limitations to the study. First, the sample size was relatively small for both groups, although adequate for qualitative analysis, which may have meant that the target populations were not adequately represented. The staff participants were of mixed professional backgrounds, which meant that adequate representation of all professional backgrounds was not possible. The patient participants were predominantly men and forcibly detained therefore perspectives from women and voluntarily admitted patients were not captured. Finally, this research was conducted within one NHS trust, which has a lower bed base than the national average and may limit the broad applicability of the findings.

In conclusion, in-patients experiencing psychosis prioritise overcoming social loss, trauma, intra- and interpersonal distress and want more control and collaboration in their treatment. A cultural and religious framework was also important to integrate into in-patient care provision. This is in some conflict with the priorities of the in-patient staff. Psychiatric in-patient care requires adaptations to ensure the priorities and needs of current inpatients are met. 
Lisa Wood BSC, MRes, DClinPsy, PhD, Lecturer in Clinical Psychology and Specialist Care Pathway Lead for Acute and Inpatient Psychology, Goodmayes Hospital, North East London Foundation Trust; School of Health and Social Care, University of Essex; and Division of Psychiatry, University College London, UK; Claire Williams, MA, DClinPsy, Strategic Lead for Acute and Inpatient Psychology and Consultant Clinical Psychologist, Goodmayes Hospital, North East London Foundation Trust, UK; Jo Billings, MA, MSC, MA MSC. DClinPSy, CPsychol, Senior Clinical Lecturer and Consultant Clinical Psychologist, MRCPsych DM, Professor of Social and Community Psychiatry, Division of Psychiatry, University College London, UK

Correspondence: Lisa Wood, Acute and Rehabilitation Directorate, Goodmayes Hospital, North East London NHS Foundation Trust, Barley Lane, Ilford IG3 8XJ, UK. Email: lisawood3@nhs.net

First received 19 Jun 2018, final revision 22 Mar 2019, accepted 24 Apr 2019

\section{Funding}

L.W. was in part supported by the National Institute for Health Research (NIHR) Collaboration for Leadership in Applied Health Research and Care (CLAHRC) North Thames at Bart's Health NHS Trust. The views expressed are those of the author(s) and not necessarily those of the NHS, the NIHR or the Department of Health and Social Care.

\section{Acknowledgements}

Thank you to all participants who made the time to participate in this study.

\section{References}

1 Yi Chong H, Teoh SL, Wu DB, Kotirum S, Chiou C, Chaiyakunapruk N. Global economic burden of schizophrenia: a systematic review. Neuropsychiatr Dis Treat 2016; 12: 357-73.

2 Ritsner MS. Handbook of Schizophrenia Spectrum Disorders, Volume III: Therapuetic Approaches, Comorbidity and Outcomes. Springer, 2011.

3. van der Post LF, Peen J, Visch I, Mulder CL, Beejman ATF, Dekker JJM. Patient perspectives and the risk of compulsory admission: the Amsterdam Study of Acute Psychiatry V. Int J Soc Psychiatry 2014; 60: 125-33.

4 Priebe S, Badesconyi A, Fioritti A, Hansson L, Kilian R, Torres-Gonzales F, et al. Reinstitutionalisation in mental health care: comparison of data on service provision from six European countries. Br Med J 2005; 330: 123-6.

5 Schoevaerts K, Bruffaerts R, Mulder CL, Vandenberghe J. An increase of compulsory admissions in Belgium and the Netherlands: an epidemiological exploration [in Dutch]. Tijdschrift voor Psychiatrie 2013; 55: 45-55.

6 Kings Fund. NHS Hospital Beds Numbers: Past, Present, Future. Kings Fund, 2017

7 Bowers L, Chaplin R, Quirk A, Lelliot P. A conceptual model of the aims and function of acute inpatient psychiatry. J Ment Health 2009; 18: 316-25.

8 Wood L, Alsawy S. Patient experiences of psychiatric inpatient care: a systematic review of qualitative evidence. J Psychiatr Intensive Care 2016; 12: $35-43$
9 Francis Report. Report of the Mid Staffordshire NHS Foundation Trust Public Enquiry. The Stationary Office, 2013.

10 Independent Mental Health Taskforce. The Five Year Forward View Mental Health Taskforce: Public Engagement Findings. Independent Mental Health Taskforce, 2015.

11 Fugard AJB, Potts HWW. Supporting thinking on sample sizes for thematic anaIyses: a quantitative tool. Int J Soc Res Methodol 2015; 18: 669-84.

12 QSR International. NVivo Qualitative Data Analysis Software Version 11. QSR International Pty Ltd, 2017.

13 Braun V, Clarke V. Using thematic analysis in psychology. Qual Res Psychol 2006; 3: 77-101.

14 Shelvin D, Houston JE, Dorahy MJ, Adamson G. Cumulative traumas and psychosis: an analysis of the National Comorbidity Survey and the British Psychiatric Morbidity Survey. Schizophr Bull 2008; 34: 193-199.

15 Read J, Fosse R, Moskowitz A, Perry B. The traumageneic neurodevelopmental model of psychosis. Neuropsychiatry 2014; 4: 65-79.

16 Heffernan S, Neil S, Weatherhead S. Religion in inpatient mental health: a narrative review. Ment Health Rev J 2014; 19: 221-36.

17 British Psychological Society. Understanding Psychosis and Schizophrenia. British Psychological Society, 2014

18 Pitt L, Kilbride $M$, Nothard S, Welford M, Morrison AP. Researching recovery from psychosis: a user-led project. Psychiatry Bull 2007; 31: 55-60.

19 Leamy M, Bird V, LeBoutillier C, Williams J, Slade M. Conceptual framework for personal recovery in mental health: systematic review and narrative synthesis. Br J Psychiatry 2011; 199: 445-52.

20 Valenti E, Giacco D, Katasakou C, Priebe S. Which values are important for patients during involuntary treatment? A qualitative study with psychiatric inpatients. Law Ethics Med 2014; 40: 832-6.

21 Kings Fund. Briefing: Mental Health Under Pressure. Kings Fund, 2015.

22 Raine R, Wallace I, Bhaird CN, Xanthopoulou P, Lanceley A, Clark A, et al. Improving the Effectiveness of Multidisciplinary Team Meetings for Patients With Chronic Diseases: A Prospective Observational Study. NIHR, 2014.

23 Giacco D, Mavromara L, Gamblen J, Conneely M, Priebe S. Shared decisionmaking with involuntary hospital patients: a qualitative study of barriers and facilitators. BJPsych Open 2018; 4: 113-8.

24 Kisely S, Wyder M, Dietrich J, Robinson G, Siskind D, Crompton D. Motivational aftercare planning to better care: applying the principles of advanced directives and motivational interviewing to discharge planning for people with mental illness. Int J Ment Health Nurs 2017; 26: 41-8.

25 Muskett C. Trauma-informed care in inpatient mental health settings: a review of the literature. Int J Ment Health Nurs 2014; 23: 51-54.

26 Thomas J, Harden A. Methods for the thematic synthesis of qualitative research in systematic reviews. BMC Med Res Methodol 2008; 8: 1-10. 
The importance of considering social circumstances and trauma

Overwhelming social issues

1. 'Well, what happened was I lost my job and I had to get rid of everything because I couldn't afford to keep it all, I think that's what's triggered it all off . I couldn't sleep. I couldn't eat. It just went downhill from there...I haven't been successful since [in gaining employment].' (Participant 2)

Trauma and grief contributing to the crisis

2. 'Why I'm here this time? I've had boys that want to throw acid on my face, they said they were going to hold me down and when they see me on the street they're going to throw acid on me...' (Participant 5)

Incorporating cultural, religious and spiritual ways of understanding mental health

3. 'They [medical professionals] are saying you can't recover if they [spiritual voices] keep bothering me, so how am I supposed to recover if they keep bothering me? ... but they're my friends, and I don't want to turn my back on them.' (Participant 1)

4. 'I mean I just prayed, I think it was on the sunday night can I have some answers Lord, I really like my life, I'm really enjoying my life, I've got, I don't want to lose these things.' (Participant 9)

Involving family and friends with agreement

5. 'My brother he always kicks off with me. He's a police officer so he always grabs me, and colludes with the family to get me brought into hospital or whatever, and I end up in hospital.' (Participant 3)

6. 'And my mum and my brother actually took me to hospital. So I was

banging my head, I was getting angry, I was trying to kill myself, and that's how I admitted myself.' (Participant 4)
Managing the intra- and interpersonal impact of psychosis. Distressing and commanding voices causing harm

7. 'The voices started telling me to do things, actually do things, talking to me, do that, do this. If you do that you're going to. .. all of a sudden it started like that. And I started.. I deteriorated. I was banging my head and getting very angry . the voices were telling me to . sort of . to kill myself, actually. They were telling me to kill myself, to end my life.' (Participant 4)

aranoia and distressing unusual beliefs - needing to protect myseff 8 . 'I remember that, because one time I was unreal and I had to cut my sk out to become real. It's kind of, you have to cut...you have to make all these marks, the army make you do it.' (Participant 1)

9. 'Disorientated when I first came in I was paranoid as well. I said don't put me round there I don't like my room, and I was like all who's that and who are these people? I was paranoid of the people' (Participant 11).

\section{Coping with my crisis the best way I can}

10. 'The heavy drinking started was when I was in the turbulent relationship, and it was to block it out. While I was sober I couldn't deal with all the

thoughts going through my head. I could lose myself in four or five pints of lager, maybe six, maybe seven pints depending. And I used to drink fast as well. I wanted to get there quick so I could forget.' (Participant 1)

11. 'I cut myself off from all of my friends because I didn't know who to trust, who was by friend. So I got to the point where I'd lost it a bit.' (Participant 5). Confusion and emotional distress

12. 'The first few days are certainly sketchy. Before people would say I came to visit you, do you remember when I went, came to visit and I say no I don't I don't remember that and this time I can't remember the first few days. (Participant 10)

13. ' I just had a panic attack go through me. Like I did the other night when we went on the table out the front [on the ward]. I was sitting here and I just, don't like being here, I just had a panic attack, which started up there and went through my body like a wave of fear.' (Participant 4)
Lack of control and collaboration in care

Admission trauma and disorientation

14. 'Oh, it was horrible. It's horrible, someone coming round your house. They come with a load of police, they come round, about four doctors. It's wrong really, the way they do it.' (Participant 2)

Inflexible and unclear treatment

15. 'They got me on a whole load of **** [medication], which I asked them to put me on one medication as it never helped me, they didn't listen to me... and I don't agree with it, but I have to comply with it.' (Participant 3) 16. 'Clarification would have been good. If they'd have turned round and said right, you've now gone to this [increased observation] level because of what happened the other day .... Okay, fair enough.' (Participant 11)

Dominant pharmacological approach to treatment

17. 'There isn't anything helpful. They're just giving me tablets night and morning, that's all...' (Participant 2)

18. 'Yeah, they've given me Depakote and I'm on one tablet and I'm on $50 \mathrm{mg}$ of [unclear] and since I've been taking it I feel good.' (Participant 5) Poor patient and staff relationships

19. 'Yeah, so I did manage to get a word with him [named nurse] once. I did come up to him and say, try and have a word with him and he said "look I've got other patients, I'm not just with you".' (Participant 10)

20. 'I don't really like it, because they're [patients] all fighting all the time... I don't want to come in here and see them all fighting and arguing It gets on your nerves a bit.' (Participant 2)

\section{Flexible access to psychosocial treatments}

21. 'You have medication, you have activities. The other thing I think you could have is something like talk time, and art time, and more activities.' (Participant 3)

22. 'I don't take part in activities.... I don't like they put thoughts in and thoughts out. So I don't want to be near them, I just stay in my room. I don't take part in activities.' (Participant 4) 
Multidisciplinary facilitators of care

\section{Team cohesiveness}

1. 'We do apply a multidisciplinary model, but I think that there are enoug distinctions within our respective roles to basically come at the same honest.' (Participant 12)

\section{Biopsychosocial treatments}

2. 'I know we have other disciplines like psychologists, we have obviously myself [occupational therapy], pharmacist. But it's definitely more nursing and medical led I would say.' (Participant 11)

Developing therapeutic relationships with patients

3. 'Everybody knows my priority's always ensuring that patients feel as if their

mornings, the first things I will do is go and speak to all the patients."

(Participant 10)

4. 'I think on this ward anyway, it's meeting the needs of someone saying want to have a chat when they want to have a chat.' (Participant 7)

\section{integrated psychological perspectives}

5. 'In short, in short what I feel psychologists are very adept at is being ab

get a proper sense of what's going on for that person and attaining an

understanding of why that might be.' (Participant 12)

6. 'I believe psychology has really

around patients.' (Participant 1)
Treating complexity and incorporating social factors

continued assessment and observation

7. ....it involves carrying out a regular review and assessment of patients with mental illness in terms of carrying out regular mental state examinations,

assessing their risks on a regular basis, making decisions around their care in the context of treatment...' (Participant 1)

8. 'Lack of insight is a key thing with psychosis anyway, ... and they're

continuously saying they don't need to be here they can become aggressive because of that...' (Participant 7)

9. 'He was here, he was discharged, an incident occurred and now he's back diagnosis. So there is some component to illicit substance use.' (Participant 6 ) management

11. 'Not going to help stigmatism at all but the main risk is violence and aggression. It is predominantly the main risk. Followed by property dama drugs and alcohol on the ward, self-harm. Those are the main risks.

(Participant 6)

12. '... when there are significant issues that need to be addressed in terms of the risk. That is when I am prepared to step up regarding medication as much as possible.' (Participant 1)

\section{Family and social network support}

13. 'Family involvement...I think family involvement, I probably have

mentioned it; feeling included and inclusive... help them to deal with issues.' (Participant 10)

14. 'Often it could be loneliness in the community, it could be like, I don't know, to be honest it could be anything it depends on the person. I think we had somebody really recently, they had a real lack of support in the

community, they were often getting involved in drugs and things.' (Participant

Social problems, functioning, and community care planning

15. 'They could be having some financial problems; it could be things like housing as well or sometimes immigration.' (Participant 4)

16. 'I would probably be more inclined to talk about the social circumstances and looking at what they're engaged in, in the community and family support. (Participant 2)

17. 'To return back to their previous level of functioning and be able to get back out there in the community and get employed, into jobs or returning back to school. Just doing the things they love to do and being back with their families and friends.' (Participant 1)

\section{Restrictive practices preventing quality care provision}

Restrictive and rigid treatment

. 'They're feeling trapped. Their liberties are being infringed. Yes. We say it's for safety they just see it as a punishment.' (Participant 5)

19. 'They will say, "Well this is my goal" - but their goal might not be, I guess, something the team would want... because we would probably see it as being a bit different or dangerous.' (Participant 10) ack of resources and confined environment

20. 'Sometimes we are too much in a haste, from diagnosis to the interventions, we are too much in a hurry, and on our acute ward, there is doctors to the nurses, we don't.' (Participant 2)

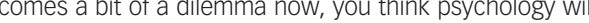
. pful for this patient and the patient hasn't been seen by the ward psychologist because the ward psychologist comes in on a Thursday [one day a week].' (Participant 1)

22. 'The psychological... stable is different for the psychological minded. You do not want somebody who is actively psychotic, it is going to be difficult for person to understand what the psychologist is about." (Participant 1 ) . The abuse is ranging from racist to all this anything that is associated with the word bad. Some spit in your face, some attack you physically, these re things you have to put up on the ward.' (Participant 2)

\section{Lack of safety}

24. 'Obviously hospital can be a scary place because dependent on how unwell you are and how aware you are of your surroundings you can be very taken aback by the shouting, the banging, the patients needing to be restrained.' (Participant 7 )

25. 'Well I think that there are possibly two schools of thought regarding that, from the staff side it is about safety, safety for that person and to others and their surroundings, because if none of those were an issue we wouldn't even be here.' (Participant 12) 